\title{
Deriving of the Generalized Special Relativity (GSR) by Using Mirror Clock and Lorentz Transformations
}

\author{
M. H. M. Hilo', R. Abd Elgani' ${ }^{1}$, R. Abd Elhai', M. D. Abd Allah', Amel A. A. Elfaki² \\ ${ }^{1}$ Department of Physics, Sudan University of Science and Technology, Khartoum, Sudan \\ ${ }^{2}$ Department of Physics, Bahri University, Khartoum, Sudan \\ Email:mahmoudhilo@gmail.com, mahmoud1972@sustech.edu, rawia abdalgani@yahoo.com, \\ rawia@sustech.edu, rashaabdelhaye@sustech.edu
}

Received 1 October 2014; revised 28 October 2014; accepted 15 November 2014

Copyright (C) 2014 by authors and Scientific Research Publishing Inc.

This work is licensed under the Creative Commons Attribution International License (CC BY).

http://creativecommons.org/licenses/by/4.0/

(c) (i) Open Access

\section{Abstract}

Einstein relativity theory shows its high capability of promoting itself to solve the long stand physical problems. The so-called generalized special relativity (GSR) was derived later, using the beautiful Einstein relation between field and space-time curvature. In this work we re-derive (GSR) expression of time by incorporating the field effect in it, and by using mirror clock and Lorentz transformations. This expression reduces to that of (GSR) the previous conventional one, besides reducing to special relativistic expression. It also shows that the speed of light is constant inside the field and is equal to $C$. This means that the observed decrease of light in matter and field is attributed to the strong interaction of photons with particles and mediates which causes successive absorption and reemission processes that lead to time delay. This absorption process makes some particles appear to move faster than light within the field or medium. This new expression, unlike that of GSR, can describe time and coordinate relativistic expressions for strong as well as weak fields at constant acceleration.

\section{Keywords}

Lorentz Transformations, Mirror Clock, Space-Time Curvature, Gravitational Field

\section{Introduction}

Einstein's special relativity and general relativity represent one of the biggest achievements that change radically the space-time concept [1]. Special relativity (SR) succeeds in explaining the constancy of light speed in va- 
cuum, long-time meson decay, and mass-energy conversion [2].

SR shows that time, length, mass, and energy are velocity dependent [3]. Einstein extends his SR theory to the so-called general relativity theory (GR) which extends the space-time interval to the curved space GR succeeded in explaining a wide variety of astronomical phenomena. These include the Doppler red shift, which is interpreted as resulting from the universe expansion, the existence of relic microwave background, beside the gravitational red shift [4].

These remarkable successes of SR and GR motivate some authors to promote SR within the frame of GR to produce the so-called Generalized Special Relativity (GSR) [5].

Mubarak model, the matter energy-momentum tenser relation on the generalized Lorentz factor $(r)$ derived from the space-time interval in the curve space, was utilized to construct the seminal EGSR model; time, length, mass and energy are dependent on the field potential as well as velocity [6].

In this work two mirrors of certain length, acting as time clock, are under the action of gravity. The motion of the two mirrors under gravity is utilized to find a useful expression of time in the presence of the gravitational field. The speed of light is assumed to be constant in the gravity field. This assumption is confirmed by obtaining the light speed in accursed space time [7].

This paper, which is concerned with the derivation of time dilation in the gravitational field, consists of 3 sections; apart from introduction, Section 2 is devoted for presenting EGSR theory. The derivation of time dilation in the gravitational field for any field is done in Section 3. The speed of light in the gravitational field is found in Section 4. Sections 5 and 6 are devoted for discussion and conclusion (Generalized Special Relativity [8]).

According to GSR the expressions of time $t$, length $L$, and mass $m$, are dependent on the velocity as well as field potential per unit mass and according to the relations.

$$
\begin{aligned}
& t=\frac{t_{0}}{\sqrt{9_{00}-v^{2} / c^{2}}} \\
& L=L_{0} \sqrt{9_{00}-v^{2} / c^{2}} \\
& m=\frac{9_{00} m_{0}}{\sqrt{9_{00}-v^{2} / c^{2}}}
\end{aligned}
$$

where

$$
9_{00}=1+\frac{2 \phi}{c^{2}}
$$

c stands for light speed in vacuum, while $L_{0}$ and $m_{0}$ represent the rest time, length and mass respectively.

\section{Time Dilation and Length Contraction}

The expression of time in the presence of gravitational field can be found by considering two as a clock, with time intervals to representing the travel between the two mirrors as shown in Figure 1.

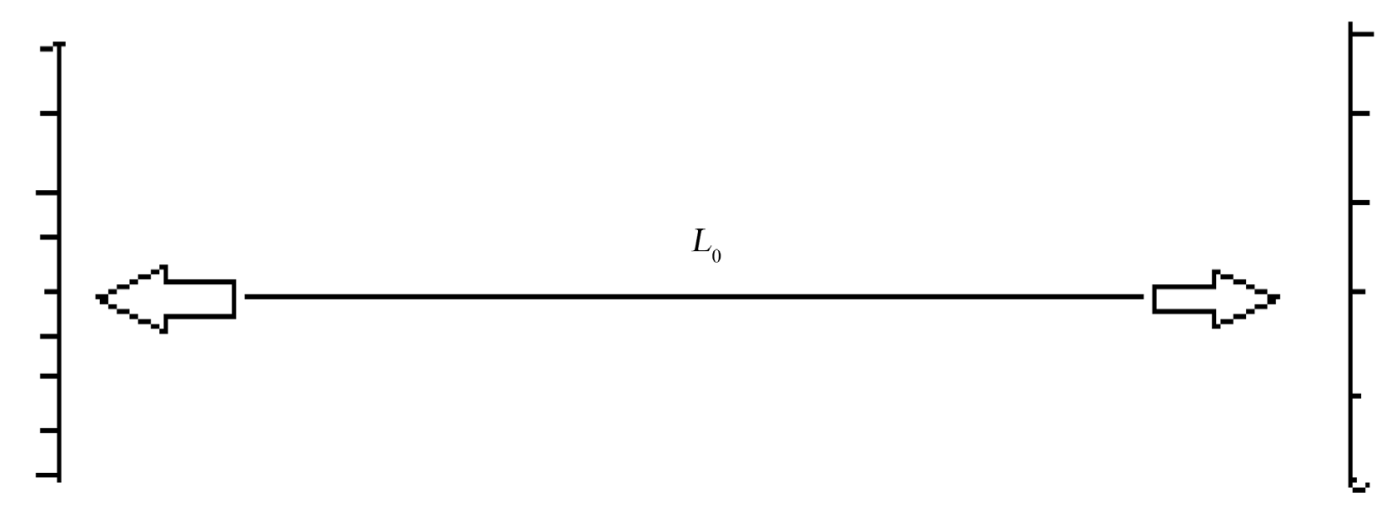

Figure 1. The mirror in free space. 


$$
L_{0}=c t_{0}
$$

It moves with velocity up ward under gravity of acceleration g, the velocity of the lower mirror is given by

$$
v^{2}=v_{0}^{2}-2 a y
$$

where $v_{0}$ stands for the initial velocity, while $y$ represents the displacement.

The vertical displacement $y$ is given by

$$
y=v_{0} t-\frac{1}{2} a t^{2}
$$

The speed $v$ is also given by $v=v_{0}-a t$

Thus

$$
y=\frac{\left(2 v_{0}-a t\right) t}{2}=\frac{\left(v_{0}+v_{0}-a t\right) t}{2}=\frac{\left(v_{0}+v\right) t}{2}=v_{a} t
$$

where: $v_{a}=\frac{\left(v_{0}+v\right)}{2} \rightarrow(8)$ is the average speed; $a$ is any arbitrary acceleration in general. For the force $F$ the potential $v$ is given by

$$
F=m a, \quad V=m a y=m \phi
$$

where $\phi$ is the potential per unit mass and is given to be

$$
\phi=a y=g y
$$

Inserting (9) in (6) yields as shown in Figure 2.

$$
v^{2}=v_{0}^{2}-2 \phi
$$

Since the point $p$ at which the photon hits the mirror is displaced $y$ meter vertically down ward, it follows that the light photon hits $p$ as shown in Figure 3.

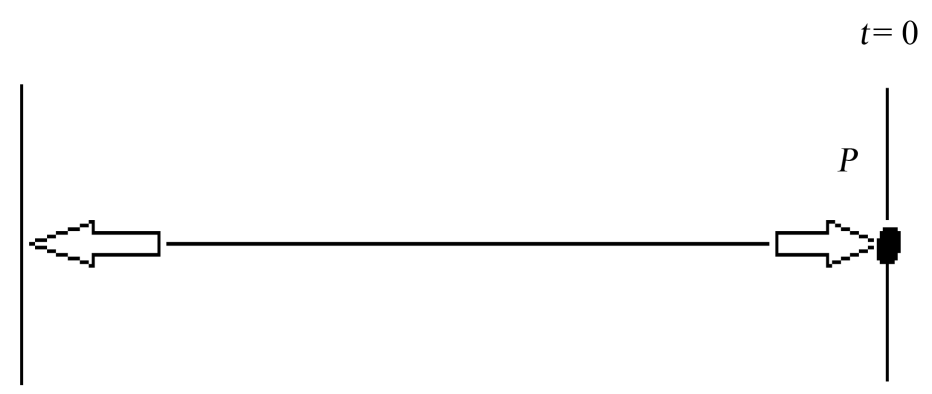

\section{Figure 2. The mirror at $t=0$.}

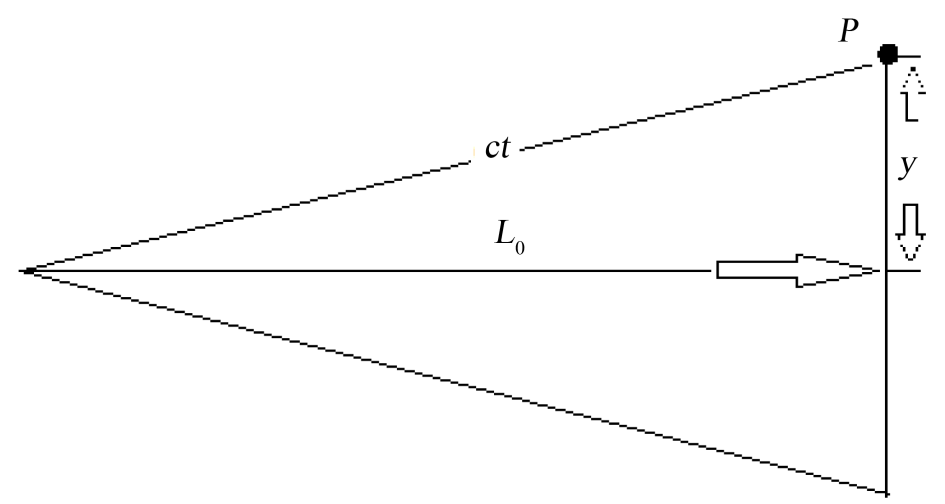

Figure 3. The mirror displaced $y$ meter vertically down ward. 
Speed $c$ in the gravitational field the distance travelled by light is given indicates that

$$
(c t)^{2}=L_{0}^{2}+y^{2}
$$

In view of Equations (7)-(10), one gets

$$
\begin{gathered}
c^{2} t^{2}=c t_{0}^{2}+v_{m}^{2} t^{2} \\
\left(1-\frac{v_{m}^{2}}{c^{2}}\right) t^{2}=t_{0}^{2} \\
t=\frac{t_{0}}{\sqrt{1-\frac{v_{a}^{2}}{c^{2}}}}=\frac{t_{0}}{\sqrt{1-\frac{\left(v_{0}+v\right)^{2}}{4 c^{2}}}}=\gamma t_{0}
\end{gathered}
$$

Thus:

$$
\gamma=\frac{1}{\sqrt{1-\frac{v_{a}^{2}}{c^{2}}}}=\frac{1}{\sqrt{1-\frac{\left(v_{0}+v\right)^{2}}{4 c^{2}}}}
$$

Using relation (10)

$$
\begin{aligned}
& \gamma=\frac{1}{\sqrt{1-\frac{v_{0}^{2}+2 v v_{0}+v^{2}}{4 c^{2}}}}, \\
& \sqrt{1-\frac{\left(2 v_{0}^{2}-2 \phi+2 v_{0} \sqrt{v_{0}^{2}-2 \phi}\right)}{4 c^{2}}}
\end{aligned}
$$

If one consider $v$ and $v_{0}$ as standing for effective speed which is related to the maximum speed through the relations

$$
v_{0}=\frac{1}{\sqrt{2}} v_{0_{\max }}, \quad v=\frac{1}{\sqrt{2}} v_{\max }
$$

Then relation (10) becomes

$$
v_{m}^{2}=v_{0_{\max }^{2}}^{2}-4 \phi
$$

And relation (13) becomes

$$
\gamma=\frac{1}{\sqrt{1-\frac{\left(2 v_{0_{\max }}^{2}-4 \phi+2 v_{0_{\max }} \sqrt{v_{0_{\max }}^{2}-4 \phi}\right)}{4 c^{2}}}}
$$

For weak field

$$
2 v_{0}\left(v_{0_{\max }^{2}}^{2}-4 \phi\right)^{\frac{1}{2}}=2 v_{0_{\max }^{2}}^{2}\left(1-\frac{4 \phi}{v_{0_{\max }}^{2}}\right)^{\frac{1}{2}} \approx 2 v_{0_{\max }^{2}}^{2}-4 \phi
$$

Thus

$$
\gamma=\frac{1}{\sqrt{1-\frac{\left(2 v_{0_{\max }^{2}}^{2}-4 \phi+2 v_{0_{\max }^{2}}^{2}-4 \phi\right)}{4 c^{2}}}}=\frac{1}{\sqrt{1+\frac{2 \phi}{c^{2}}-\frac{v_{0_{\max }^{2}}^{2}}{c^{2}}}}
$$


Which coincides completed with the expression of time in the presence of the gravitational field obtain within the framework of GSR. Relation (14) it is important to note that of the factor $1 / 2$ in Einstein SR energy expression where

$$
E=\frac{1}{2} m v_{m}^{2}=m\left(\frac{v_{m}}{\sqrt{2}}\right)^{2}=m v^{2}
$$

According to Equations (9) and (10) this relation holds for any field other than the gravitational field.

The length contraction can be obtained by considering a clock falling by sliding on a rod of a height $L_{0}$. For the observer moving with a clock the average speed is given by

$$
v=\frac{L}{t_{0}}
$$

where the rod is moving and accelerated w.r.t him, thus his length is $L$. But for an observer at rest w.r.t the rod, the rod length is $L_{0}$ for him and the clock time is it. Thus the speed $v$ is given by

$$
v=\frac{L_{0}}{t}
$$

Thus, with the aid of Equations (18) (19) and (13).

$$
\begin{aligned}
& \frac{L}{t_{0}}=\frac{L_{0}}{t} \\
& L=L_{0} \frac{t_{0}}{t}=\gamma^{-1} L_{0}
\end{aligned}
$$

This is in agreement with the corresponding expression in GSR.

\section{Derivation of Time and Coordinate Expressions by Using Lorentz Transform}

Using Lorentz transformation, the event at point $\left(x_{1}, t_{1}\right)$ in the frame $x$ at a point $x$ is given to be according to Equation (7) by replacing $y$ by $x$

$$
x=\gamma\left(x^{\prime}+v_{a} t^{\prime}\right)
$$

Since the space is homogeneous it follows that

$$
x^{\prime}=\gamma\left(x+v_{a} t\right)
$$

Consider ampoule of light emitted from a source when the origins 0 and 01 are in coincidence at

$$
t=t^{\prime}=0
$$

In this care

$$
\begin{aligned}
& x=c t \\
& x^{\prime}=c t^{\prime}
\end{aligned}
$$

Substituting Equation (23) in Equations (22) and (21) yields

$$
\begin{aligned}
& c t=\gamma\left(c t^{\prime}+v_{a} t^{\prime}\right)=\gamma\left(c+v_{a}\right) t^{\prime} \\
& c t^{\prime}=\gamma\left(c t-v_{a} t\right)=\gamma\left(c-v_{a}\right) t
\end{aligned}
$$

Inserting Equation (25) in Equation (24) yields

$$
\begin{gathered}
c t=\frac{\gamma^{2}}{c}\left(c+v_{a}\right)\left(c-v_{a}\right) t \\
c^{2}=\gamma^{2}\left(c^{2}-v_{a}^{2}\right) t
\end{gathered}
$$




$$
\gamma=\frac{1}{\sqrt{1-\frac{v_{a}^{2}}{c^{2}}}}
$$

With the aid of Equation (8) and Equation (10)

$$
\gamma=\frac{1}{\sqrt{1-\frac{\left(v_{0}^{2}+2 v v_{0}+v^{2}\right)}{4 c^{2}}}}=\frac{1}{\sqrt{1-\frac{\left(v_{0}^{2}-\phi+v_{0} \sqrt{v_{0}^{2}-2 \phi}\right)}{2 c^{2}}}}
$$

When one consider the expression for the maximum speed in Equation (15), one gets

$$
\gamma=\frac{1}{\sqrt{1-\frac{\left(v_{0_{\max }}^{2}-2 \phi+v_{0_{\max }} \sqrt{v_{0_{\max }^{2}}^{2}-4 \phi}\right)}{2 c^{2}}}}
$$

It is very striking to observe that when no field exist, i.e. $\phi=0$

$$
\gamma=\frac{1}{\sqrt{1-\frac{v_{0_{\max }^{2}}^{c^{2}}}{c^{2}}}}
$$

This is the usual SR expression.

For weak field

$$
\frac{\phi}{v_{0_{\max }}^{2}} \prec \prec 1
$$

Therefore

$$
\left(v_{0_{\max }}^{2}-4 \phi\right)^{\frac{1}{2}}=v_{0_{\max }}\left(1-\frac{2 \phi}{v_{0_{\max }}^{2}}\right)
$$

Thus Equation (25) becomes

$$
\gamma=\sqrt{1-\frac{\left(v_{0_{\max }^{2}}^{2}-2 \phi+v_{0_{\max }^{2}}^{2}-2 \phi\right)}{2 c^{2}}}
$$

This is the usual expression of GSR for a weak field.

\section{Discussion}

In Section 3 time dilation expression in the presence of any field has been derived by using mirror, see Equations (11) and (14). The only assumption which causes a limitation to this expression is the constancy of acceleration. The mirror motion is described by using Newton's Law of linear motion with constant acceleration.

The speed of light is assumed to be constant. The resulting expression for $t$ reduced to that of SR in the absence of a field as shown by Equation (16) where

$$
\gamma=\frac{1}{\sqrt{1-\frac{v_{0_{\max }^{2}}^{2}}{c^{2}}}}
$$

It also reduced to GSR form for a weak field as shown in Equation (16).

The expression of length contraction has been obtained as well. Again this expression reduced to SR and GSR also [9].

Lorentz transformation is also utilized to find a relativistic expression for $t$ and $x$. The expression shown in 
Equations (27)-(29) indicates that these expressions are similar to that obtained by the mirror method and are reduced to the corresponding SR and GSR expressions.

\section{Conclusion}

The expressions for time and length obtained by using Lorentz transformation and using mirror clock, for fields at constant acceleration, and by assuming the speed of light to be constant indicate that GSR rests on a solid ground. It also indicates that space and time are affected by any field, not gravity only. Unlike the curved spacetime derivation, where the field is assumed to be week, this derivation holds for strong fields as well. By reducing to SR and GSR for a weak field, it indicates its self-consistency.

\section{References}

[1] Lawdre, D.F. (1982) An Introduction to Tensor Calculus and Relativity. John Wiley and Sons, New York, Chapter 5, 6.

[2] Weinberg, S. (1972) Gravitation and Cosmology. John Wiley and Sons, New York, Chapter 5, 6.

[3] Savickas, D. (2002) Relation between Newtonian Mechanics, General Relativity and Quantum Mechanics. Journal of Physics, 70, 798-807.

[4] Mubarak, D.A. and Ali, T. (2003) The Special Relativity in the Presence of Gravitation and Other Field. University of Khartoum, Khartoum.

[5] Dirrar, M., Hilo, M.H.M., Abd Elgani, R. and Bakheet, A.M.A. (2013) Neutrino Speed Can Exceed the Speed of Light within the Frame Work of the Generalized Special Relativity and Savickas Model. Natural Science, 5, 685-688. http://dx.doi.org/10.4236/ns.2013.56084

[6] Hilo, M.H.M. (2011) Using of the Generalized Special Relativity in Deriving the Equation of the Gravitational Red-Shift. Journal of Modern Physics, 2, 370-373. http://dx.doi.org/10.4236/jmp.2011.25045

[7] Hilo, M.H.M., et al. (2011) Using of the Generalized Special Relativity (GSR) in Estimating the Neutrino Masses to Explain the Conversion of Electron Neutrinos. Natural Science, 3, 334-338. http://dx.doi.org/10.4236/ns.2011.34044

[8] Narlikar, J.V. (1993) Introduction to Cosmology. 2nd Edition, Cambridge University Press, Cambridge.

[9] Xu, C., Uis, G.F.R.E., Xu, X.J. and El Tahir, A. (1992) The Generalized Field Equation. South African Journal of Physics, 15, 5.7. 
Scientific Research Publishing (SCIRP) is one of the largest Open Access journal publishers. It is currently publishing more than 200 open access, online, peer-reviewed journals covering a wide range of academic disciplines. SCIRP serves the worldwide academic communities and contributes to the progress and application of science with its publication.

Other selected journals from SCIRP are listed as below. Submit your manuscript to us via either submit@scirp.org or Online Submission Portal.
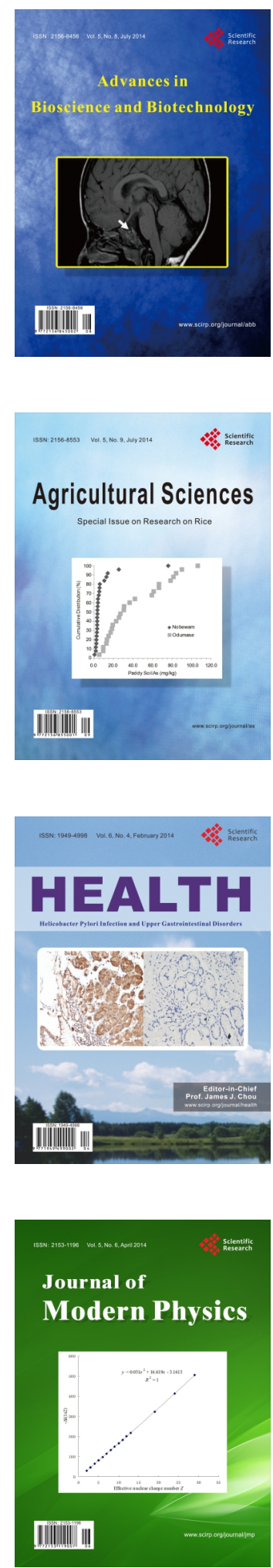
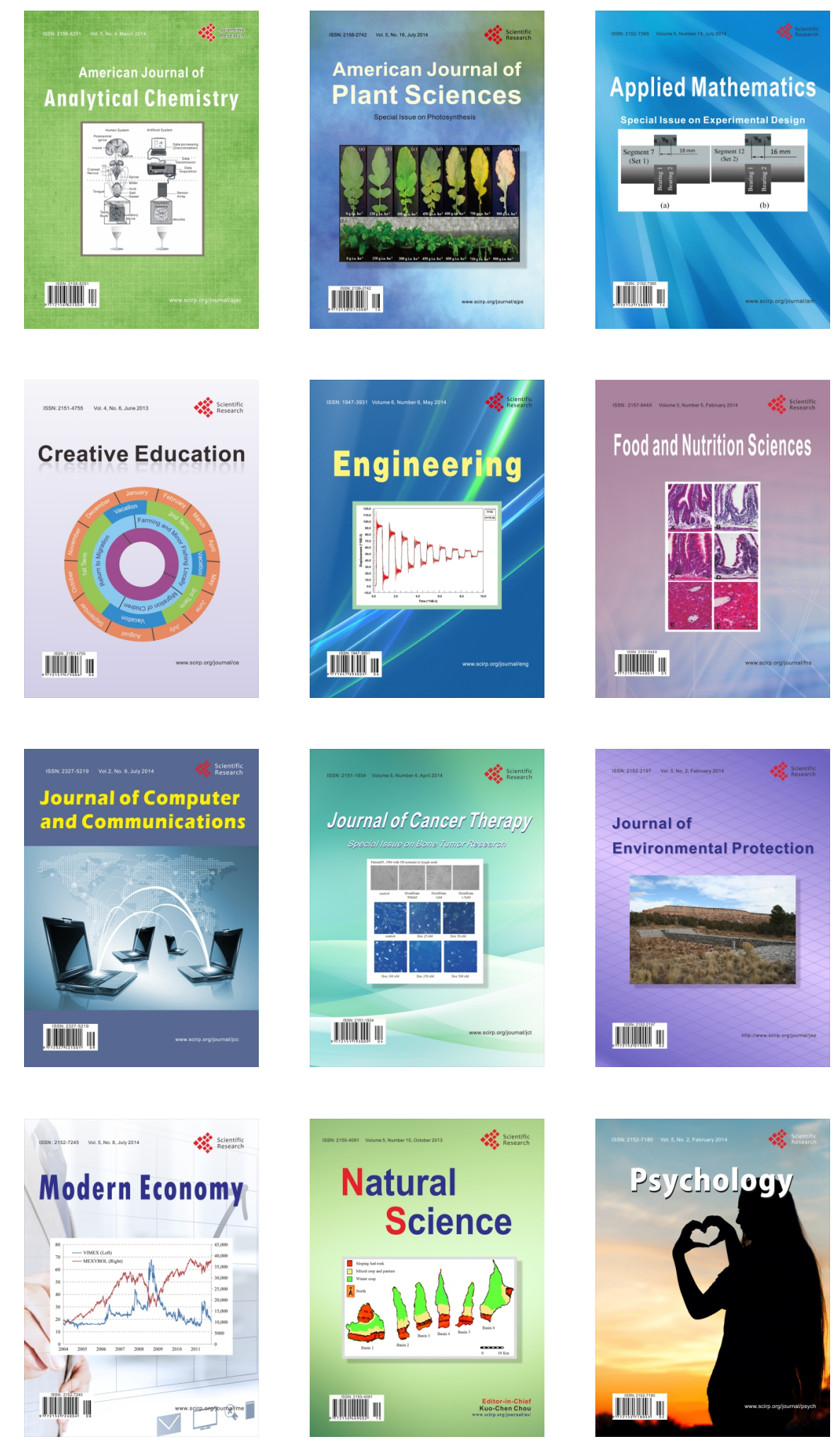